\title{
STAT3-targeting RNA interference inhibits pancreatic cancer angiogenesis in vitro and in vivo
}

\author{
CHEN HUANG $^{1,2,3^{*}}$, TAO JIANG ${ }^{1 *}$, LIN ZHU $^{1}$, JUN LIU $^{1,2,3}$, JUN CAO $^{1}$, \\ KE-JIAN HUANG ${ }^{1}$ and ZHENG-JUN QIU ${ }^{1,2,3}$
}

\author{
${ }^{1}$ Department of General Surgery, Affiliated First People's Hospital, Shanghai Jiao Tong University, \\ 200080 Shanghai; ${ }^{2}$ Shanghai Key Laboratory of Pancreas Disease, 200080 Shanghai; ${ }^{3}$ Pancreatic \\ Cancer Center of Shanghai Jiaotong University, 200080 Shanghai, P.R. China
}

Received January 3, 2011; Accepted March 22, 2011

DOI: 10.3892/ijo.2011.1000

\begin{abstract}
Signal transducers and activators of transcription 3 (STAT3) is a central cytoplasmic transcription factor that is activated by phosphorylation in response to extracellular signals and oncogenes. STAT3 regulates a number of pathways important in tumorigenesis including cell cycle progression, apoptosis, tumor angiogenesis, invasion and metastasis, and tumor cell evasion of the immune system. Our studies demonstrated that constitutively activated STAT3 plays an important role in the angiogenesis of pancreatic cancer. The objective of this study was to evaluate the potential use of RNA interference (RNAi) to knock down the STAT3 gene and the effect on angiogenesis of human pancreatic cancer cells in vitro and in vivo. We stably inhibited the expression of STAT3 and phosphorylated STAT3 (p-STAT3) using RNAi in the SW1990 pancreatic cancer cell line. Furthermore, RNAi for STAT3 inhibited STAT3-induced HUVEC cell migration and cell proliferation, and significantly suppressed the levels of secreted vascular endothelial growth factor (VEGF) and matrix metalloproteinases-2 (MMP-2) of SW1990 cells. In vivo experiments showed that RNAi for STAT3 significantly suppressed tumor growth and angiogenesis of SW1990 cells. Furthermore, silencing the STAT3 gene in SW1990 cells by RNAi also led to a decrease of VEGF and MMP-2 at the mRNA and protein levels. Collectively, these results demonstrate that the STAT3 signaling pathway plays an important role in the angiogenesis of pancreatic cancer and that knockdown of the STAT3 gene using the RNAi technique may be a novel therapeutic option for the treatment of pancreatic cancer.
\end{abstract}

Correspondence to: Dr Zhengjun Qiu, Shanghai Jiao Tong University, Affiliated First People's Hospital, Department of General Surgery, 100 Haining Road, 200080 Shanghai, P.R. China

E-mail: qiuwryb@online.sh.cn

* Contributed equally

Key words: signal transducers and activators of transcription 3, RNAi, pancreatic cancer, angiogenesis

\section{Introduction}

Pancreatic cancer is the fourth most common cause of adult cancer death, accounting for an estimated 42,470 new cases and 35,240 deaths in USA for 2009 (1). The high mortality rate is due to the aggressive biological properties, late onset of symptoms and the failure of systemic therapies (2). Of all the treatments used against pancreatic cancer, radical surgery is still the only one that can completely eradicate this disease (3). However, in only $5-25 \%$ of the patients presenting with pancreatic cancer will the tumor be operable. Even after curative resection, the actual 5-year survival is only $10-20 \%$ (4). Unfortunately, effective systemic therapy capable of reversing the aggressive nature of this disease is currently not available and the specific molecular regulatory pathways involved in pancreatic cancer initiation and progression have not been fully characterized.

Angiogenesis plays important roles in many developmental and pathological processes including tumor growth and metastasis. It has been well established that all successful tumors must undergo neovascularization, or angiogenesis, in order to acquire nutrients for continued growth and metastatic spread. Without angiogenesis, a solid tumor rarely grows larger than 2 to $3 \mathrm{~mm}(5)$.

Abundant angiogenesis regulators and activation of oncogene are essential for an angiogenesis phenotype that supports tumorigenicity (6). Although numerous angiogenesis regulators or oncogenes are involved, signal transducers and activators of transcription 3 (STAT3) play a pivotal role in tumor angiogenesis (7-9). STAT3, a member of the signal transduction and activation of transcription family, is a key cytoplasmic transcription factor activated by tyrosine kinase growth factor and cytokine receptors. Once tyrosine is phosphorylated, two STAT3 monomers form dimers through reciprocal phospho-tyrosine-SH2 interactions, and translocate to the nucleus where they bind to STAT3-specific DNA-response elements of angiogenesis-related target genes, and induce angiogenesis-related target gene transcription $(10,11)$.

Many researchers have proved that the angiogenesis-related gene such as vascular endothelial growth factor (VEGF) and matrix metalloproteinases-2 (MMP-2) are the target genes of STAT3 and STAT3 and could promote tumor angiogenesis and metastasis through upregulating the expression of VEGF (7-9) 
and MMP-2 $(12,13)$. Our previous studies demonstrated that overexpression of STAT3, p-STAT3, VEGF and MMP-2 was found in pancreatic carcer and the expression of p-STAT3 was positively correlated with VEGF and MMP-2 (14-16). Taken together, these data indicate that STAT3 is associated with angiogenesis and metastasis through upregulating angiogenesisrelated genes in pancreatic cancer, and inhibition of the expression or function of STAT3 may improve the disease outcome.

The present study was designed to evaluate the potential use of RNA interference (RNAi) to knock down STAT3 expression and activation and the effect on the angiogenesis of human pancreatic cancer cells in vitro and in vivo. The angiogenesis phenotypic changes resulting from the reduction of STAT3 expression were studied both in vitro and in vivo. We found that knockdown of the STAT3 gene by RNAi significantly suppressed the expression of VEGF and MMP-2, which was accompanied by marked inhibition of STAT3 induced HUVEC cell migration and cell proliferation in vitro and tumor cell angiogenesis in vivo. Our results demonstrate that STAT3 signaling pathway plays important roles in angiogenesis of pancreatic cancer and that knockdown of STAT3 gene using RNAi technique may be a novel therapeutic option for treatment of pancreatic cancer.

\section{Materials and methods}

Cell lines and culture conditions. Human pancreatic cancer cell line SW1990 was purchased from American Type Culture Collection. They were maintained in Dulbecco's modified Eagle's medium (DMEM) supplemented with $10 \%$ fetal calf serum, 100 units $/ \mathrm{ml}$ penicillin, and $100 \mu \mathrm{g} / \mathrm{ml}$ streptomycin in a humidified incubator with an atmosphere of $5 \% \mathrm{CO}_{2}-95 \%$ air at $37^{\circ} \mathrm{C}$. Human umbilical vein endothelial cells (HUVEC) were purchased from American Type Culture Collection. They were maintained in DMEM supplemented with $10 \%$ fetal calf serum, $2 \mathrm{mmol} / \mathrm{l}$ glutamine, HAT (hypoxanthine $0.1 \mathrm{mmol} / \mathrm{l}$, aminopterin $0.4 \mathrm{mmol} / \mathrm{l}$, thymidine $16 \mathrm{mmol} / \mathrm{l}), 100 \mathrm{units} / \mathrm{ml}$ penicillin, and $100 \mu \mathrm{g} / \mathrm{ml}$ streptomycin in a humidified incubator with an atmosphere of $5 \% \mathrm{CO}_{2}-95 \%$ air at $37^{\circ} \mathrm{C}$.

Transfection. In our previous studies, we constructed three STAT3 specific siRNA expression vectors (pRNAT-STAT3siRNA-I, II, III) and proved pRNAT-STAT3 siRNA-II had the most obvious gene silencing effect (17). We also constructed a negative control scrambled siRNA expression vector (pRNAT-Con). For stable transfection, SW1990 cells were transfected with pRNAT-STAT3-RNAi-II plasmid which had the most obvious gene silencing effect. SW1990 cells were also transfected with the pRNAT-Con vector. Cells were then selected with a standard medium containing $500 \mathrm{mg} /$ ml G418 (Gibco, USA) for 14 days. pRNAT-Con and pRNATSTAT3-RNAi-II expression vector were carried out in SW1990 cells, and G418-resistant colonies were pooled to establish stable SW1990 pRNAT-Con transfectants (SW1990-Con) and SW1990 STAT3-RNAi transfectants (SW1990-RNAi). The stable transfection cells were then used for subsequent studies.

Cell migration assay. Transwells (Costar, Cambridge, MA) were pretreated with serum-free medium at $37^{\circ} \mathrm{C}$ for $1 \mathrm{~h}$ before seeding with HUVECs at $1 \times 10^{5}$ per well in $100 \mu$ l endothelial basal medium with $0.1 \%$ fetal bovine serum. The transwells were then inserted into 24 -well plates containing $600 \mu \mathrm{l}$ conditioned medium and incubated at $37^{\circ} \mathrm{C}$ for $6 \mathrm{~h}$ to allow HUVEC cells to migrate. Cells on the upper side of the filter were removed with cotton swabs. Migrated cells on the lower side of the filter were fixed and stained with HE. The number of migrated cells was counted under a binocular microscope.

Cell proliferation assay. Cultured supernatants from SW1990, SW1990-Con and SW1990-RNAi cells were collected. The metabolic activity of HUVEC cells was determined by methyl thiazoleterazolium (MTT) assay. Briefly, exponentially growing HUVEC cells $\left(2.5 \times 10^{5}\right)$ were seeded in 96-well culture plates in culture medium at an optimal density. After $24 \mathrm{~h}$, the medium was changed to conditioned medium from SW1990, SW1990-Con and SW1990-RNAi cells. MTT assays were performed after 1,2 and 3 days of conditioned medium treatment. At the time of the assay, the cells were stained with $20 \mu \mathrm{l}$ MTT $\left(5 \mathrm{mg} / \mathrm{ml}\right.$ ) (Sigma, USA) at $37^{\circ} \mathrm{C}$ for $4 \mathrm{~h}$ and subsequently solubilized in $150 \mu \mathrm{l}$ of DMSO. OD readings were obtained at $570 \mathrm{~nm}$. The cell growth rate was represented by the relative ratio of OD570 at 24, 48, and $72 \mathrm{~h}$, to OD570 at $0 \mathrm{~h}$, respectively. The growth curve was drawn according to the cell growth rate.

Cell cycle assay. HUVEC were collected and fixed after $48 \mathrm{~h}$ culture with conditioned medium from SW1990, SW1990-Con and SW1990-RNAi cells. After incubation in RNase A for $30 \mathrm{~min}$ at $37^{\circ} \mathrm{C}$, the cells were stained with propidium iodide (PI). Flow cytometric analysis was done using a FACScan instrument (Becton-Dickinson, Mountain view, CA) and CellQuest software.

Enzyme-linked immunosorbent assay (ELISA). SW1990, SW1990-Con and SW1990-RNAi cells were seeded into 24-well plates. Fresh medium was added after overnight culture. The cultured supernatants were collected $24 \mathrm{~h}$ later and centrifuged to eliminate cellular fragments. VEGF and MMP-2 protein accumulated in the culture medium were analyzed using sandwich ELISA, wherein the supernatant of the culture was incubated with VEGF antibody (goat polyclonal anti-human VEGF, Santa Cruz, USA), MMP-2 antibody (goat polyclonal anti-human MMP-2, Santa Cruz, USA) and streptavidin alkaline phosphatase (Santa Cruz). The antigen-antibody complex was then incubated with p-nitrophenyl phosphate (Sigma) and dissolved in pNPP buffer (Chemicon, USA). MMP-2 and VEGF concentrations in the samples were determined from the absorbance at $570 \mathrm{~nm}$ spectrophotometrically.

Eggs and animals. Fertile eggs were acquired from Merial Vital Laboratory Animal Technology Company (Beijing, China). After washing the outsides of the shells with soap and saturating in a 70\% ethyl alcohol bath for $30 \mathrm{sec}$, the fertile eggs were incubated in an incubator with an atmosphere of $5 \% \mathrm{CO}_{2}-95 \%$ air at $37^{\circ} \mathrm{C}$ and $60 \%$ relative humidity and rotated $180^{\circ}$ three times daily. Male athymic BALB/c nude mice were obtained from the Animal Center of Chinese Academy of Science (Shanghai, China) and housed in laminar flow cabinets under specific pathogen-free conditions. The mice were used when they were 6-8 weeks old. The use of eggs and animals in this study complies with the Guide for the Care and Use of Laboratory Animals (NIH publication no. 86-23, revised 


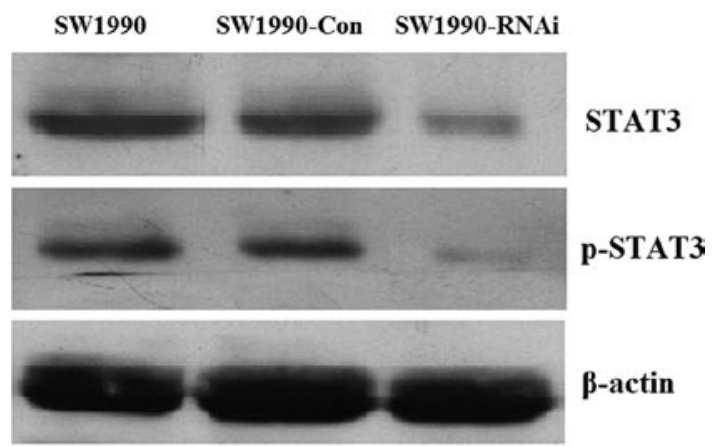

Figure 1. Effect of stable transfection of pRNAT-STAT3-siRNA-II vector on STAT3 expression. Western blot analysis. Cellular whole and nuclear protein extracts (100 $\mu \mathrm{g}$ in each) were prepared from SW1990 cells, SW1990 cells transfected with a control vector (SW1990-Con), and SW1990 cells transfected with STAT3-RNAi (SW1990-RNAi). The expression of STAT3 protein was determined using Western blot analysis with an anti-STAT3 antibody. The expression of p-STAT3 protein was determined using Western blot analysis with an anti-p-STAT3 antibody. The levels of $\beta$-actin expression were determined as a control for equivalent protein loading.

A

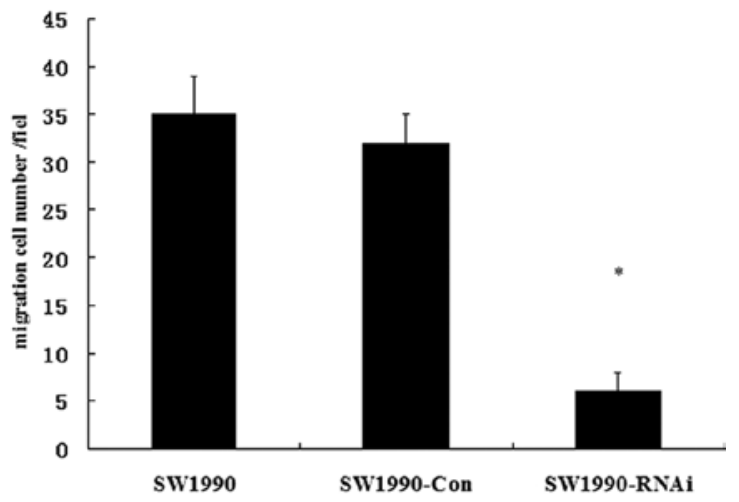

B

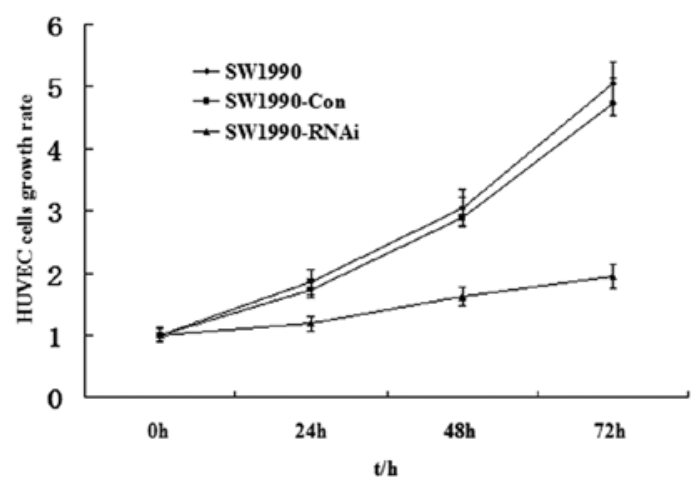

Figure 2. Effect of STAT3 small interfering RNA on STAT3 induced HUVEC cell migration and cell proliferation. (A) HUVEC cell migration was quantified after $6 \mathrm{~h}$, few HUVEC cells migrated as the chemotactic stimulus from SW1990-RNAi cells supernatants compared with SW1990 and SW1990-Con cells supernatants. (B) HUVEC cells were incubated with the indicated supernatant were subjected to MTT assay as described in Materials and methods. The HUVEC cell proliferation was reduced significantly after treatment with SW1990-RNAi conditioned supernatant $(\mathrm{P}<0.05)$ as compared with that of SW1990 or SW1990-Con conditioned supernatant.

1985) and the current Chinese regulations and standards on the use of Laboratory Animals.
Chick embryo chorioallantoic membrane (CAM) assay. The CAM model used in these studies was carried out according to the procedure described by Storgard et al (18) and Xu et al (19). In brief, eggs were candled using a hand held-egg candler at the blunt end of the egg to identify the air sac and prominent blood vessels after incubating for 9 days. Using a mini drill, the CAM was separated from the shell by making a shallow burr hole at the blunt end on the egg and another burr hole made perpendicular to the previously identified blood vessels in the center of the egg. Mild suction was applied to the blunt end burr hole to displace the air sac and drop the CAM away from the shell. Fine forceps were then used to pick away the shell over the air sac, so that a window could be made and the CAM identified. SW1990, SW1990-Con and SW1990-RNAi cells (in a total volume of $100 \mu 1,1.0 \times 10^{7}$ cells) were transplanted on the CAM in an avascular area under sterile conditions. The window was sealed with sterile scotch tape and the egg returned to the incubator. On day 9 of inoculation, the tapes were removed and digital images of the live CAM were captured using a dissection microscope and a colour video camera. Next, the tumor volume ( $a^{2} \times b x 0.4$, where a represents the longer axis and $b$ represents the shorter axis) was measured (19).

In vivo tumorigenicity assay. Six-to-eight-week-old male athymic BALB/c nude mice were housed in laminar flow cabinets under specific pathogen-free conditions. SW1990 cells and stable transfection cells (SW1990-Con, SW1990-RNAi) were injected into the right flank of mice in a total volume of $100 \mu \mathrm{l}\left(1.0 \times 10^{7}\right.$ cells). The tumor-bearing mice were sacrificed 35 days after inoculation and the tumor were removed and weighed. Then, the tumor tissue was fixed in formalin, embedded in paraffin, cut into $5 \mu \mathrm{m}$ sections, and stained with $\mathrm{HE}$.

Microvessel counting. Tissue sections stained with CD34 were used for evaluating MVD. Immunohistochemical staining was performed as follows: formalin-fixed paraffin-embedded tissue sections of $5 \mu \mathrm{m}$ thickness were dewaxed in $100 \%$ xylene and rehydrated by serial incubations in 100, 90 and $80 \%$ ethanol, followed by phosphate-buffered saline (PBS). Antigen retrieval was performed by microwaving slides in $10 \mathrm{mM}$ sodium citrate buffer ( $\mathrm{pH}$ 6.0) for $1 \mathrm{~min}$ at full power followed by $9 \mathrm{~min}$ at medium power, according to the manufacturer's instructions. To inactivate the endogenous peroxidase activity, sections were treated with freshly prepared $0.3 \%$ (vol/vol) hydrogen peroxide in methanol in dark, for $30 \mathrm{~min}$, at room temperature (RT). Non-specific antibody binding was then blocked with 5\% goat serum in PBS for $90 \mathrm{~min}$ at RT. The sections were then incubated with rabbit anti-human CD34 polyclonal antibody (Santa Cruz) or rabbit polyclonal IgG controls (Vector Laboratories, USA) in blocking buffer overnight at $4^{\circ} \mathrm{C}$. The sections were then rinsed in wash buffer (PBS containing $0.5 \%$ bovine serum albumin, $0.1 \%$ Tween-20) and incubated for 30 min with biotinylated goat anti-rabbit IgG (ABC staining kit, Santa Cruz) diluted according to the manufacturer's protocol. Next, a solution of avidin-conjugated horseradish peroxidase (ABC staining kit) was applied for $30 \mathrm{~min}$, according to the manufacturer's instruction. Peroxidase activity was developed in $0.5 \%$ (vol/vol) 3,3'-diaminobenzidine hydrochloride (DAB, Sigma) in PBS containing $0.03 \%$ (vol/vol) hydrogen peroxide for $2 \mathrm{~min}$. Sections were counterstained with Harris' hematoxylin 


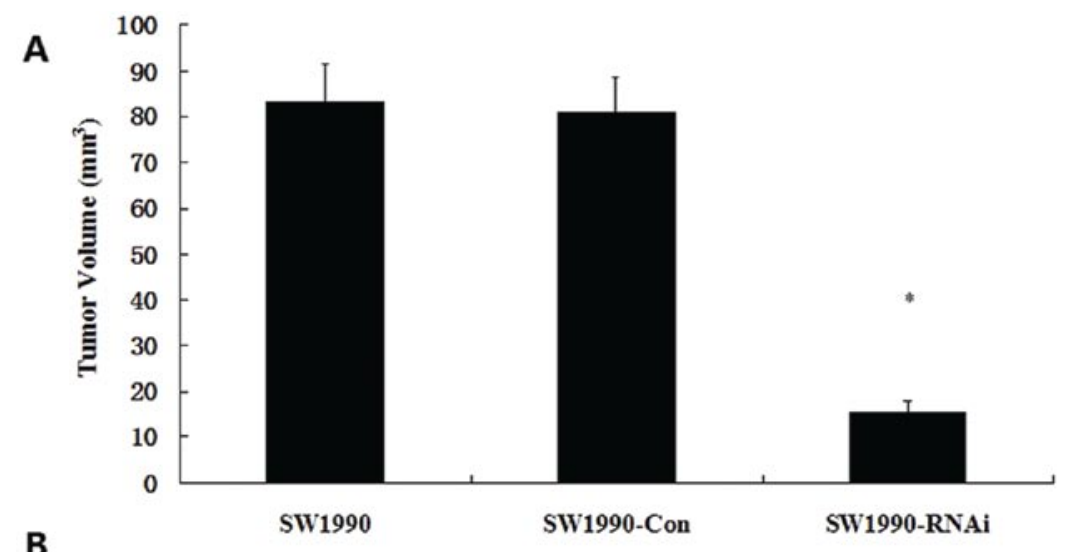

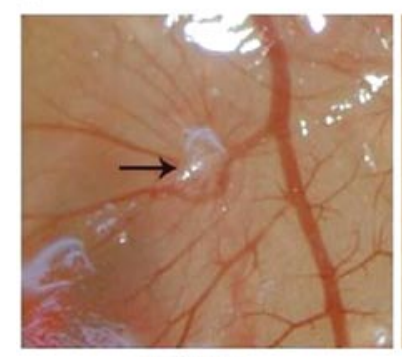

SW1990

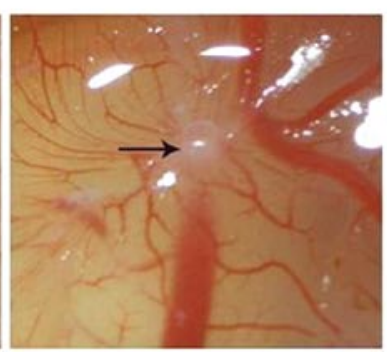

SW1990-Con

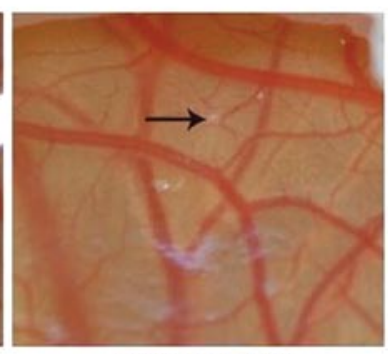

SW1990-RNAi

Figure 3. Effect of STAT3 small interfering RNA on panreatic cancer growth and angiogenesis in CAM model. (A) SW1990, SW1990-Con and SW1990-RNAi cells were transplanted on the CAM in an avascular area under sterile conditions. All fertile eggs (six of six) developed tumors from parental SW1990 cells or SW1990-Con cells without significant difference in tumor volume. In contrast, only four of six fertile eggs developed tumors from SW1990-RNAi cells and the tumors were significantly smaller than those of control eggs $(\mathrm{P}<0.05)$. (B) Tumors from SW1990 and SW1990-Con cells induced abundant neovessels around tumor body. On the contrary, when the SW1990-RNAi cells were transplanted on the CAM, a robust decrease in neovessel around tumor body was observed. The tumor nodes are shown by arrows.

and mounted in gelatin (Sigma, USA). Then the slides were examined under x100 magnification for the hot spots rich in vessels and MVDs were counted under x400 magnification, so that every single brown-stained cell and cell cluster was calculated as a blood vessel, regardless of whether a vessel lumen structure was seen. Five different fields were chosen on each of the slides, and the stained vessels were counted simultaneously by 2 researchers under a microscope. The average of the 5 areas was recorded as the MVD score (20).

$R T-P C R$. Total RNA extraction from tumor cells was performed with TRIzol Reagent (Life Technologies, USA). Then, $2 \mu \mathrm{g}$ of total RNA were reverse-transcribed with the First Strand cDNA Synthesis kit (Promega, USA) to synthesize cDNA samples. Subsequently, $2 \mu 1$ of cDNA product was then subjected to PCR amplification with Taq DNA polymerase (Sangon, China) on a thermal cycler using the following primers. The oligo-nucleotide primers for STAT3 were constructed under the help of primerdesign software 'Primer Premier 5.0'. The oligo-nucleotide primers for MMP-2, VEGF and $\beta$-actin were constructed on the basis of the published sequence. The PCR primers used to detect each factor were as follows: VEGF, sense strand 5'-CCTGGT GGACATCTTCCAGGAGTACC-3', antisense strand 5'-GAA GCTCATCTCTCCTATGTGCTGGC-3', with a product length of 196 bp (21); MMP-2, sense strand 5'-GTGCTGAAGGAC ACACTAAAGAAGA-3', antisense strand 5'-TTGCCATCCT TCTCAAAGTTGTAGG-3', with a product length of $605 \mathrm{bp}$ (22); $\beta$-actin, sense strand 5'-ATCTGGCACCACACCTTCT ACAATGAGCTGCG-3', antisense strand 5'-CGTCATACTC
CTGCTTGCTGATCCACATCTGC-3', with a product length of 838 bp (23). The PCR conditions were as follows: one cycle of denaturing at $94^{\circ} \mathrm{C}$ for $5 \mathrm{~min}$, followed by 30 cycles of $94^{\circ} \mathrm{C}$ for $1 \mathrm{~min}, 60^{\circ} \mathrm{C}$ for $1 \mathrm{~min}$ and $72^{\circ} \mathrm{C}$ for $1 \mathrm{~min}$, before a final extension at $72^{\circ} \mathrm{C}$ for $10 \mathrm{~min}$. The PCR products were loaded onto $2 \%$ agarose gels and visualized with ethidium bromide under UV light. This experiment was performed three times and representative data are shown.

Western blot analysis. Whole-cell protein extracts and Nuclear protein extracts from tumor cells were prepared with RIPA Lysis Buffer (Santa Cruz, USA) and Nuclear Extract kit (Active Motif), according to the manufacturer's instructions, respectively. Protein concentrations were determined using a Bio-Rad assay kit (Bio-Rad, USA). Lysates containing $100 \mu \mathrm{g}$ of protein were mixed with loading-buffer with $5 \% \beta$-mercaptoethanol, and heated for $5 \mathrm{~min}$ at $100^{\circ} \mathrm{C}$. Samples were separated by sodium dodecyl sulfate-polyacrylamide gel electrophoresis (SDS-PAGE) and transferred onto nitrocellulose membranes by semi-dry blotting. Membranes were incubated in blocking buffer (1X TBS, $0.1 \%$ Tween 20 , and $5 \%$ non-fat dry milk) for $1 \mathrm{~h}$ at room temperature, followed by hybridization with anti-p-STAT3 [tyr-705] antibody (Cell Signal, USA, 1:1000 dilution), antiSTAT3 antibody (Cell Signal, 1:1000 dilution), anti-VEGF antibody (Santa Cruz, 1:1000 dilution), anti-MMP-2 antibody (Santa Cruz, 1:000 dilution) or anti- $\beta$-actin antibody (Labvision, USA, 1:100 dilution), at $4^{\circ} \mathrm{C}$ overnight. After three washes in TBS $/ 0.1 \%$ Tween 20 , the membranes were hybridizated with a horseradish peroxidase-conjugated secondary antibody 


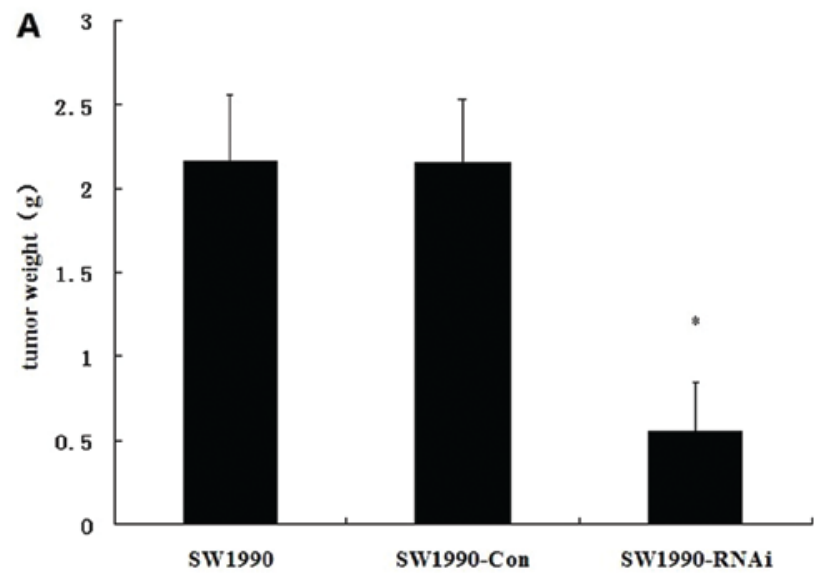

B

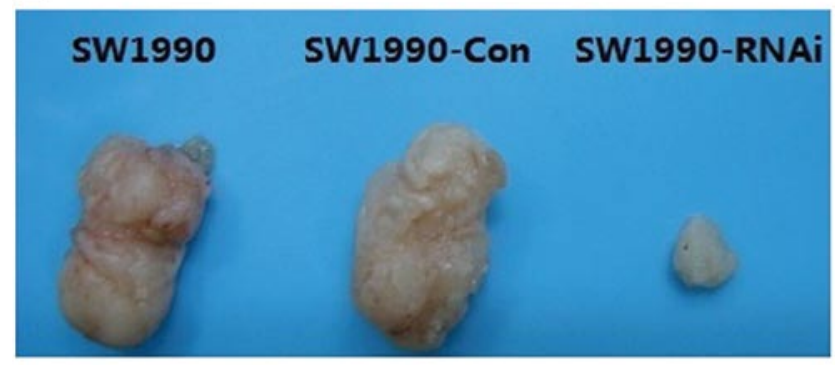

Figure 4. Effect of STAT3 small interfering RNA on panreatic cancer growth and angiogenesis in a nude mouse model. (A) SW1990, SW1990-Con and SW1990-RNAi cells were inoculated s.c. into nude mice. All mice (six of six) developed tumors from parental SW1990 cells or SW1990-Con cells without significant difference in tumor weight. In contrast, only three of six mice developed tumors from SW1990-RNAi cells and the tumors were significantly smaller than those of control mice $(\mathrm{P}<0.05)$. (B) Tumors from SW1990 and SW1990-Con cells appeared highly vascularized. On the contrary, when the SW1990-RNAi cells were implanted in vivo, a robust decrease in vascularization was observed.

rabbit $\operatorname{IgG}$ (Santa Cruz, 1:5000 dilution) for $1 \mathrm{~h}$ at room temperature. After three washes in TBS/0.1\% Tween 20, signals were detected by chemiluminescence using the Western Blotting Luminol Reagent (Santa Cruz, USA). The experiments were performed three times and representative data are shown.

\section{Results}

Silencing of STAT3 expression by stable transfection of STAT3 specific shRNA expression vector. Total and nuclear protein extracts were prepared from parental SW1990, pRNAT-Con and pRNAT-STAT3-siRNA-II transfected cells, and the levels of STAT3 and p-STAT3 protein expression in these cells were determined by Western blotting. Densitometric measurements indicated that the levels of STAT3 and p-STAT3 protein expression in the transfectants containing pRNAT-STAT3siRNA-II (SW1990-RNAi) were decreased 92.3 and $90.1 \%$ as compared to parental SW1990 cells (Fig. 1). The result indicated that table transfection of pRNAT-STAT3-siRNA-II vector silenced STAT3 expression.

Effect of STAT3 small interfering RNA on STAT3 induced HUVEC cell migration, cell proliferation and cell cycle. We collected cultured supernatants from SW1990, SW1990-Con and SW1990-RNAi cells. As shown in Fig. 2A, the culture supernatants from SW1990 and SW1990-Con cells induced robust endothelial cell migration. By contrast, few endothelial cells migrated when conditioned medium from SW1990-RNAi cells was used as the chemotactic stimulus.

To determine the effect of STAT3 siRNA on STAT3 induced HUVEC cell proliferation, the metabolic activity of HUVEC cells treated with SW1990, SW1990-Con and SW1990-RNAi conditioned medium was quantified by MTT. The HUVEC cell proliferation was reduced significantly after treatment with SW1990-RNAi conditioned medium $(\mathrm{P}<0.05)$ as compared with that of SW1990 or SW1990-Con conditioned medium (Fig. 2B).

To analyze the effect of STAT3 siRNA on STAT3 induced HUVEC cell cycle, flow cytometric analysis was applied to analyze the HUVEC cell cycle. As shown in Table I, the percentage of cells at $\mathrm{G}_{0} / \mathrm{G}_{1}$ phase was increased from $65.96 \%$ (parental SW1990) to $80.95 \%$ (SW1990-RNAi) and the S-phase cells were decreased from $16.59 \%$ (parental SW1990) to $4.57 \%$ (SW1990-RNAi).

Effect of STAT3 small interfering RNA on secreted VEGF and $M M P-2$ protein expression. ELISA was performed using human VEGF-specific antibody and MMP-2-specific antibody to quantify the amount of VEGF and MMP-2 protein in the culture media. When cells were transfected with pRNAT-STAT3siRNA-II, the VEGF concentration in the media was decreased significantly (Table II) compared with cells transfected with pRNAT-Con or parental SW1990 cells $(\mathrm{P}<0.05)$. A similar inhibitory effect on MMP-2 protein levels is shown in Table II, which demonstrated that the expression of MMP-2 protein in the culture medium of SW1990 cells was also significantly inhibited after STAT3 silencing. These results indicated that silence of STAT3 gene suppressed secreted VEGF and MMP-2 protein expression.

Effect of STAT3 small interfering RNA on panreatic cancer growth and angiogenesis in the CAM model. To determine whether inhibition of STAT3 by siRNA had an effect on tumor growth and angiogenesis, SW1990, SW1990-Con and SW1990-RNAi cells were transplanted on the CAM in an avascular area under sterile conditions. All fertile eggs (six of six) developed tumors from parental SW1990 cells or SW1990-Con cells without significant difference in tumor volume. In contrast, only four of six fertile eggs developed tumors from SW1990-RNAi cells and the tumors were significantly smaller than those of control mice (Fig. 3A). Tumors from SW1990 and SW1990-Con cells induced abundant neovessels around the tumor body. On the contrary, when the SW1990-RNAi cells were transplanted on the CAM, a robust decrease in neovessel around the tumor body was observed (Fig. 3B).

Effect of STAT3 small interfering RNA on panreatic cancer growth and angiogenesis in the nude mouse model. To determine whether inhibition of STAT3 by siRNA had an effect on tumor growth and angiogenesis, SW1990, SW1990-Con and SW1990-RNAi cells were inoculated s.c. into nude mice. The six mice (6/6) developed tumors from parental SW1990 cells or 


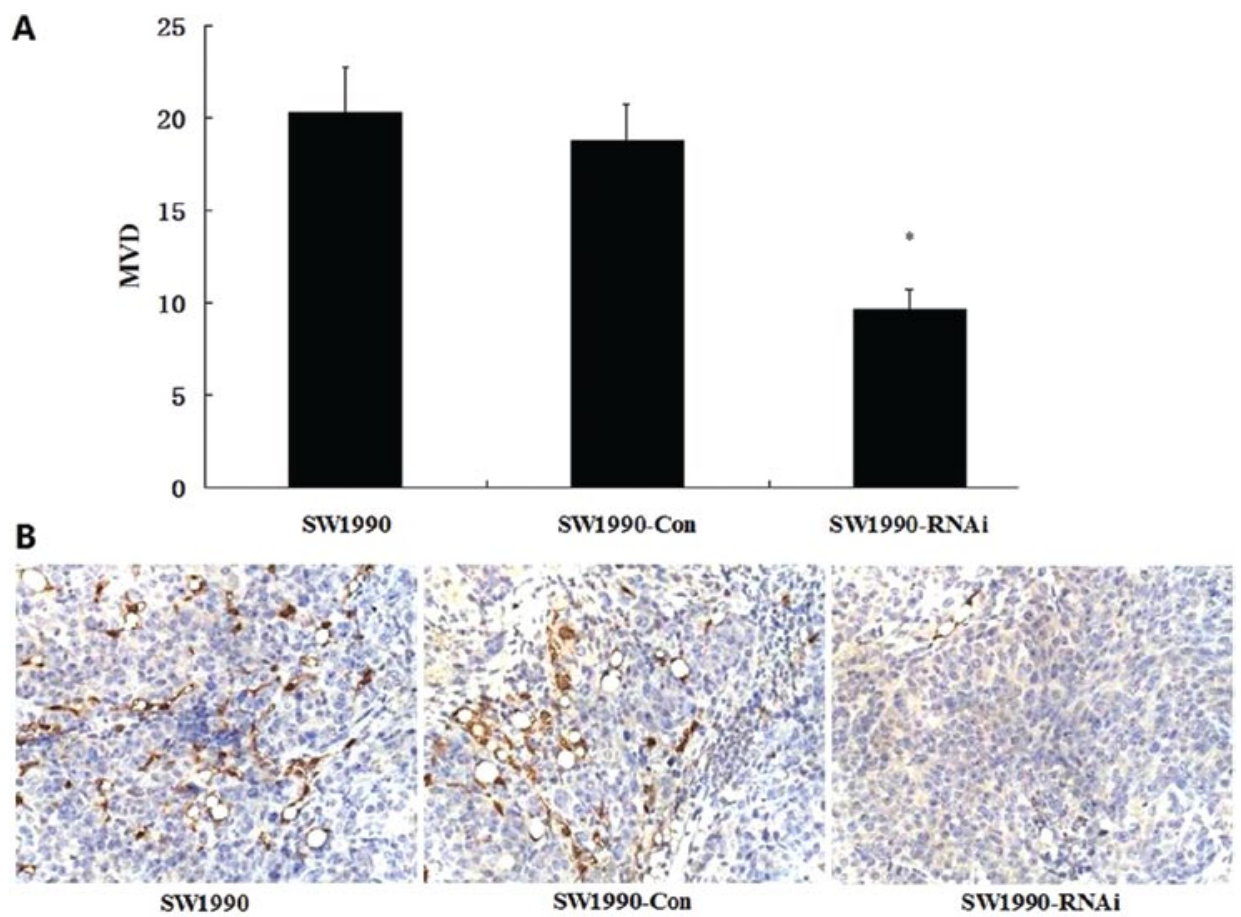

Figure 5. Effect of STAT3 small interfering RNA on panreatic cancer MVD in the nude mouse model. Tissue sections stained with CD34 was used for evaluating the MVD changes of tumors after inhibition of STAT3 in vivo. (A) MVD was significantly decreased in tumors formed by SW1990-RNAi $(\mathrm{P}<0.05)$. The MVD in tumors treated with SW1990-Con was similar to that observed in the SW1990 tumors. (B) CD34-positive vessels were abundant in SW1990 and SW1990-Con tumors. On the contrary, CD34-positive vessels were significantly decreased in tumors formed by SW1990-RNAi.

A

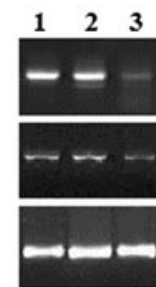

VEGF

1 : SW1990

MMP-2 2:SW1990-Con

B-actin 3 : SW1990-RNAi

B
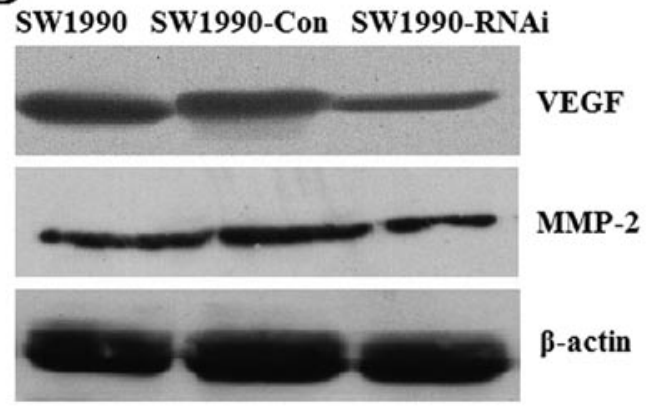

Figure 6. Effect of STAT3 small interfering RNA on the expression of VEGF and MMP-2. (A) RT-PCR analysis. The RNA samples ( $2 \mu \mathrm{g}$ in each) extracted from SW1990 cells, SW1990 cells transfected with a control vector (SW1990-Con), and SW1990 cells transfected with STAT3-RNAi (SW1990-RNAi) were subjected to RT-PCR for VEGF, MMP-2, and $\beta$-actin mRNAs as described in Materials and methods. RT-PCR for $\beta$-actin was performed in parallel to show an equal amount of total RNA in the sample. (B) Western blot analysis. Cellular whole protein extracts (100 $\mu \mathrm{g}$ in each) were prepared from SW1990 cells, SW1990 cells transfected with a control vector (SW1990-Con), and SW1990 cells transfected with STAT3-RNAi (SW1990-RNAi). The expression of VEGF protein was determined using Western blot analysis with an anti-VEGF antibody. The expression of MMP-2 protein was determined by hybridizing the same membrane filter with an anti-MMP- 2 antibody. The levels of $\beta$-actin expression were determined as a control for equivalent protein loading.
SW1990-Con cells without significant difference in tumor weight. In contrast, only three of six mice developed tumors from SW1990-RNAi cells and the tumors were significantly smaller than those of the control mice (Fig. 4A). Tumors from SW1990 and SW1990-Con cells appeared highly vascularized. On the contrary, when the SW1990-RNAi cells were implanted in vivo, a robust decrease in vascularization was observed (Fig. 4B). Next, we checked the MVD changes of tumors after inhibition of STAT3 in vivo. As shown in Fig. 5A and B, CD34positive vessels were abundant in SW1990 and SW1990-Con tumors. MVD was significantly decreased in tumors formed by SW1990-RNAi. The MVD in tumors treated with SW1990-Con was similar to that observed in the SW1990 tumors.

Effect of STAT3 small interfering RNA on VEGF and MMP-2 expression of $S W 1990$ cells. STAT3 activation contributes to oncogenesis through regulation of its target genes. To determine the effect of downregulation of STAT3 on angiogenesis-related target gene expression, we assayed for the expression of VEGF and MMP-2 by RT-PCR both of which are directly involved in tumor angiogenesis. As shown in Fig. 6A, the expression of VEGF and MMP-2 mRNAs in SW1990 cells were significantly inhibited after STAT3 silencing. The densitometric analyses revealed VEGF relative expression of SW1990-RNAi cells was reduced to $46 \%$ of that of parental SW1990 cells. And MMP-2 relative expression of SW1990-RNAi cells was reduced to $28 \%$ of that of parental SW1990 cells. A similar inhibitory effect on protein levels is shown in Fig. 6B. These results demonstrated that blockade of STAT3 signaling was able to inhibit SW1990 cell angiogenesis potential by downregulating VEGF and MMP-2. 
Table I. Effects of silence of STAT3 gene on STAT3 induced HUVEC cell cycle.

\begin{tabular}{lccc}
\hline Group & $\mathrm{G}_{0} / \mathrm{G}_{1}$ & $\mathrm{~S}$ & $\mathrm{G}_{2} / \mathrm{M}$ \\
& $(\% ;$ mean $\pm \mathrm{SD})$ & $(\% ;$ mean $\pm \mathrm{SD})$ & $17.45 \pm 1.34$ \\
\hline SW1990 & $65.96 \pm 5.52$ & $16.59 \pm 1.92$ & $15.31 \pm 1.03$ \\
SW1990-Con & $68.08 \pm 5.98$ & $16.61 \pm 1.87$ & $14.48 \pm 1.29$ \\
SW1990-RNAi & $80.95 \pm 7.49^{\mathrm{a}}$ & $4.57 \pm 0.87^{\mathrm{a}}$ & $\mathrm{mean} \pm \mathrm{SD})$ \\
\hline
\end{tabular}

${ }^{\mathrm{a}} \mathrm{P}<0.05$ compared with control.

Table II. Effects of silence of STAT3 gene on secreted VEGF and MMP-2 protein in SW1990 cells.

\begin{tabular}{lcc}
\hline Group & VEGF (pg/ml) & MMP-2 (pg/ml) \\
\hline SW1990 & $38.76 \pm 4.64$ & $29.47 \pm 3.52$ \\
SW1990-Con & $40.12 \pm 5.12$ & $26.53 \pm 3.15$ \\
SW1990-RNAi & $15.39 \pm 5.83^{\mathrm{a}}$ & $9.88 \pm 2.98^{\mathrm{a}}$ \\
\hline
\end{tabular}

${ }^{\mathrm{a}} \mathrm{P}<0.05$ compared with control.

\section{Discussion}

STAT3 is a key signal transduction protein that mediates signaling by numerous cytokines, peptide growth factors, oncoproteins and plays important roles in cancer progression $(24,25)$. Several studies have shown that STAT3 is abundantly expressed in pancreatic cancer tissues and cells $(26,27)$. In our previous studies, we demonstrated that overexpression of STAT3, p-STAT3, VEGF and MMP-2 was found in pancreatic carcer and the expression of p-STAT3 was positively correlated with VEGF and MMP-2 (14-16). In the present study, we proved that STAT3 played an important role in the angiogenesis of panreatic cancer by selectively inhibiting STAT3 expression using plasmid vector-based RNAi.

RNAi represents a key gene therapy technique in mammalian systems. Compared with traditional gene therapy methods, RNAi possesses the advantages of exquisite precision and high efficacy in downregulating gene expression, thus providing a new approach to the treatment of diseases including cancer $(28,29)$. Delivery of siRNA can be achieved through exogenous application of enzymatically or chemically synthesized siRNA or through endogenous expression using plasmid or viral vector delivery system. Enzymatically or chemically synthesized siRNA is costly and has been shown to have a relatively short half-life with only transient inhibition of the target gene because of its low transfection rate and short duration of interference. But, plasmid or viral vector-based siRNA technology has some advantages over enzymatically or chemically synthesized siRNA. Firstly, vector-based RNAi induces more efficient and stable RNA interference. Moreover, vector-based RNAi permits co-expression of reporter genes such as GFP or luciferase, which facilitates tracking and selection of transfected cells $(30,31)$.
In the present study, we used plasmid vector-based RNAi targeting STAT3 to silence the expression of STAT3 in human pancreatic cancer cells SW1990. We successfully constructed the recombinant plasmid pRNAT-STAT3-RNAi-II (14) and employed the recombinant plasmid to generate SW1990-RNAi cell line, which showed a significantly decreased STAT3 expression. Attenuation of STAT3 changed the angiogenesis behavior of human SW1990 cells in vitro and in vivo. In vitro assay revealed targeting STAT3 by RNAi inhibited STAT3 induced HUVEC cell migration, cell proliferation and arrested HUVEC cells at $\mathrm{G}_{0} / \mathrm{G}_{1}$ phase. Moreover, targeting STAT3 by RNAi inhibited tumor growth and angiogenesis of SW1990 cells in vivo with CAM and the nude mouse tumor xenograft model.

Angiogenesis is the formation of new blood vessels from pre-existing ones, which consists of several steps: endothelial cell (EC) proliferation, migration, basement membrane degradation, and new lumen organization (32). It has been demonstrated that abundant genes play important roles in tumor angiogenesis. The inhibitory mechanism in the angiogenesis after STAT3 silencing with RNAi is considered as downregulation of these genes related with tumor angiogenesis. Vascular endothelial growth factor (VEGF) is one of the most important of the known angiogenic mitogens $(33,34)$. Previous studies have demonstrated that VEGF is associated with poor survival and prognosis for pancreatic cancer. Furthermore, it has been correlated with the increase in the microvessel density (MVD), angiogenesis, local invasion and liver metastasis (35-37). A recent study has reported that VEGF is a downstream target gene of STAT3 and stable transfection of dominantnegative STAT3 (DN-STAT3) decreases VEGF expression of the pancreatic cancer cell line FG $(8,9)$. In this study, we also found that the silencing of STAT3 significantly decreased the mRNA and protein expression of VEGF in SW1990 cells. Tumor angiogenesis also needs degradation of extracellular matrix (ECM) besides angiogenic mitogens to form new blood vessels. Matrix metalloproteinases (MMPs) constitute a family of secreted, zinc-dependent endopeptidases that are required for ECM degradation in a variety of physiological and pathological tissue remodeling processes, including tumor angiogenesis, invasion and metastasis (38). Recently, some studies have found that STAT3 signaling directly regulates MMP-2 expression in melanoma cells and proved MMP-2 to be a target gene of STAT3 $(12,13)$. In our present study, the silencing of STAT3 also reduced the mRNA and protein expression of MMP-2 in SW1990 cells. 
In conclusion, the present study indicates that RNAi targeting STAT3 via a plasmid vector-based system effectively sustains the silence of STAT3 gene expression in SW1990 cells. The impaired STAT3 expression results in reduced SW1990 cell angiogenesis in vitro and in vivo through downregulation the expression of VEGF and MMP-2. Targeting STAT3 expression and activation with RNAi may be a potential therapeutic strategy in the treatment of pancreatic cancer.

\section{Acknowledgements}

This study was supported by a grant (no. 09QA1404600) awarded by fund for scientific research of Science and Technology Commission of Shanghai Municipality and a grant (No. 0801) awarded by fund for scientific research of Affiliated First People's Hospital of Shanghai Jiao Tong University. We thank Dr Wei Qiu and Dr Ming Xu for providing technical assistance.

\section{References}

1. Jemal A, Siegel R, Ward E, Hao Y, Xu J and Thun MJ: Cancer Statistics, 2009. CA Cancer J Clin 59: 225-249, 2009.

2. Pliarchopoulou K and Pectasides D: Pancreatic cancer: current and future treatment strategies. Cancer Treat Rev 35: 431-436, 2009.

3. Loos M, Kleeff J, Friess H and Buchler MW: Surgical treatment of pancreatic cancer. Ann N Y Acad Sci 1138: 169-180, 2008.

4. Yokoyama Y, Nimura Y and Nagino M: Advances in the treatment of pancreatic cancer: limitations of surgery and evaluation of new therapeutic strategies. Surg Today 39: 466-475, 2009.

5. Folkman J: Angiogenesis in cancer, vascular, rheumatoid and other disease. Nat Med 1: 27-31, 1995.

6. Volpert OV, Dameron KM and Bouck N: Sequential development of an angiogenic phenotype by human fibroblasts progressing to tumorigenicity. Oncogene 14: 1495-1502,1997.

7. Wei LH, Kuo ML, Chen CA, Chou CH, Lai KB, Lee CN and Hsieh CY: Interleukin-6 promotes cervical tumor growth by VEGF-dependent angiogenesis via a STAT3 pathway. Oncogene 22: 1517-1527, 2003.

8. Niu G, Wright KL, Huang M, Song L, Haura E, Turkson J, Zhang S, Wang T, Sinibaldi D, Coppola D, Heller R, Ellis LM, Karras J, Bromberg J, Pardoll D, Jove R and Yu H: Constitutive Stat 3 activity up-regulates VEGF expression and tumor angiogenesis. Oncogene 21: 2000-2008, 2002.

9. Wei D, Lei X, Zheng L, Wang L, Frey JA, Gao AC, Peng Z, Huang S, Xiong HQ, Abbruzzese JL and Xie K: Stat 3 activation regulates the expression of vascular endothelial growth factor and human pancreatic cancer angiogenesis and metastasis. Oncogene 22: 319-329, 2003

10. Darnell JE Jr: STATs and gene regulation. Science 277: 1630-1635, 1997.

11. Bowman T, Garcia R, Turkson J and Jove R: STATs in oncogenesis. Oncogene 19: 2474-2488, 2000.

12. Xie TX, Wei D, Liu M, Gao AC, Ali-Osman F, Sawaya R and Huang S: Stat 3 activation regulates the expression of matrix metalloproteinase- 2 and tumor invasion and metastasis. Oncogene 23: 3550-3560, 2004.

13. Xie TX, Huang FJ, Aldape KD, Kang SH, Liu M, Gershenwald JE, Xie K, Sawaya R and Huang S: Activation of Stat3 in Human Melanoma Promotes Brain Metastasis. Cancer Res 66: 3188-3196, 2006.

14. Liu C, Qiu ZJ, Sun HC, Hu HH and Huang KJ: Expressions of pSTAT3 and VEGF in pancreatic carcinoma and its clinical significances. Chinese J Hepatob Surg 12: 471-473, 2006.

15. Qiu ZJ, Huang C, Liu J, Jiang T, Zhu L, Zhang F, Cao J and Huang KJ: Correlation between STAT3 signal pathway and expression of MMP-2 in pancreatic cancer. Chinese J Gen Surg 22: 932-935, 2007

16. Huang C, Qiu ZJ, Sun J, Chen G, Chai XJ, Hu HH and Cao J: Expressions of p-STAT3 and MMP-2, MMP-9 in pancreatic cancer and their clinical significances. Chinese J Hepatob Surg 13: 851-853, 2007.
17. Qiu ZJ, Huang C, Sun J, Jiang T, Huang KJ, Cao J and Zhang F: RNAi-mediated STAT3 gene silencing inhibits invasion and metastasis of human pancreatic cancer cells. Cancer Sci 98: 1099-1106, 2007.

18. Storgard C, Mikolon D and Stupack DG: Angiogenesis assays in the chick CAM. Methods Mol Biol 294: 123-136, 2005.

19. Xu M, Jiang HB and Wang XP: Establishment of an implanted model of human pancreatic carcinoma on chick embryo and study of its tumor biology. Chinese J Pancreat 2: 101-104, 2006.

20. Maeda K, Chung YS, Ogawa Y, Takatsuka S, Kang SM, Ogawa M, Sawada T and Sowa M: Prognostic value of vascular endothelial growth factor expression in gastric marcinoma. Cancer 77: 858-863, 1996.

21. Brown KJ, Maynes SF, Bezos A, Maguire DJ, Ford MD and Parish CR: A novel in vitro assay for human angiogenesis. Lab Invest 75: 539-555, 1996.

22. Uchima Y, Sawada T, Nishihara T, Maeda K, Ohira M and Hirakawa K: Inhibition and mechanism of action of a protease inhibitor in human pancreatic cancer cells. Pancreas 29: 123-131, 2004.

23. Zhu Z, Yao J, Wang F and Xu Q: TNF-alpha and the phenotypic transformation of human peritoneal mesothelial cell. Chin Med J (Engl) 115: 513-517, 2002.

24. Haura EB, Turkson J and Jove R: Mechanisms of disease: Insights into the emerging role of signal transducers and activators of transcription in cancer. Nat Clin Pract Oncol 2: 315-324, 2005.

25. Huang S:Regulation of metastases by signal transducer and activator of transcription 3 signaling pathway: Clinical implications. Clin Cancer Res 13: 1362-1366, 2007.

26. Scholz A, Heinze S, Detjen KM, Peters M, Welzel M, Hauff P, Schimer M, Wiedenmann B and Rosewicz S: Activated signal transducer and activator of transcription 3 (STAT3) supports the malignant phenotype of human pancreatic cancer.Gastroenterology 125: 891-905, 2003.

27. Huang C, Cao J, Huang KJ, Zhang F, Jiang T, Zhu L and Qiu ZJ: Inhibition of STAT3 activity with AG490 decreases the invasion of human pancreatic cancer cell in vitro. Cancer Sci 97: 1417-1423, 2006.

28. Leung RK and Whittaker PA: RNA interference: from gene silencing to gene-specific therapeutics. Pharmacol Ther 107: 222-239, 2005.

29. Uprichard SL: The therapeutic potential of RNA interference. FEBS Lett 579: 5996-6007, 2005.

30. Allen D, Kenna PF, Palfi A, McMahon HP, Millington-Ward S, O'Reilly M, Humphries P and Farrar GJ: Development of strategies for conditional RNA interference. J Gene Med 9: 287-298, 2007.

31. Best A, Handoko L, Schluter E and Goringer HU: In vitro synthesized small interfering RNAs elicit RNA interference in african trypanosomes: an in vitro and in vivo analysis. J Biol Chem 280: 20573-20579, 2005.

32. Tandle A, Blazer DG III and Libutti SK: Antiangiogenic gene therapy of cancer: recent developments. J Transl Med 2: 22, 2004.

33. Grunstein J, Roberts WG, Mathieu-Costello O, Hanahan D and Johnson RS: Tumor-derived expression of vascular endothelial growth factor is a critical factor in tumor expansion and vascular function. Cancer Res 59: 1592-1598, 1999.

34. Millauer B, Shawver LK, Plate KH, Risau W and Ullrich A: Glioblastoma growth inhibited in vivo by a dominant-negative Flk-1 mutant. Nature 367: 576-579, 1994.

35. Itakura J, Ishiwata T, Friess H, Fujii H, Matsumoto Y, Buchler MW and Korc M: Enhanced expression of vascular endothelial growth factor in human pancreatic cancer correlates with local disease progression. Clin Cancer Res 3: 1309-1316, 1997.

36. Seo Y, Baba H, Fukuda T, Takashima M and Sugimachi K: High expression of vascular endothelial growth factor is associated with liver metastasis and a poor prognosis for patients with ductal pancreatic adenocarcinoma. Cancer 88: 2239-2245, 2000.

37. Niedergethmann M, Hildenbrand R, Wostbrock B, Hartel M, Sturm JW, Richter A and Post S: High expression of vascular endothelial growth factor predicts early recurrence and poor prognosis after curative resection for ductal adenocarcinoma of the pancreas. Pancreas 25: 122-129, 2002.

38. Ray JM and Stetler-Stevenson WG: The role of matrix metalloproteases and their inhibitors in tumour invasion, metastasis and angiogenesis. Eur Respir J 7: 2062-2072, 1994. 